\title{
An Update on the Management of Pediatric Obstructive Sleep Apnea
}

\author{
Benjamin J. Rubinstein, $M D^{1}$ \\ Cristina M. Baldassari, MD, FAAP, FACS ${ }^{1,2, *}$
}

\author{
Address \\ ${ }^{1}$ Department of Otolaryngology Head and Neck Surgery, Eastern Virginia Medical \\ School, Norfolk, VA, USA \\ *,2Department of Pediatric Otolaryngology, The Children's Hospital of The King's \\ Daughters, Norfolk, VA, USA \\ Email: cristina.baldassari@chkd.org
}

Published online: 5 July 2015

(C) Springer International Publishing AG 2015

This article is part of the Topical Collection on Otolaryngology

Keywords Sleep apnea $\cdot$ Pediatrics $\cdot$ Adenotonsillectomy $\cdot$ Obesity $\cdot$ Positive airway pressure $\cdot$ Tonsillar hypertrophy

\section{Opinion statement}

Obstructive sleep apnea (OSA) is a sleep-related breathing disorder characterized by episodes of upper airway collapse during sleep. The severity of OSA is categorized according to the apnea hypopnea index (AHI) found on polysomnogram. Pediatric OSA is classified as mild (AHI 1-5), moderate (AHI 5-10), or severe (AHI $>10)$. While the exact pathophysiology of OSA is still being investigated; adenotonsillar hypertrophy is the most common cause of obstruction in children. Thus, adenotonsillectomy is the mainstay of treatment for moderate to severe OSA. Patients at high risk for perioperative respiratory complications, including those less than 3 years of age and those with significant co-morbid medical conditions like Trisomy 21, should be monitored overnight following surgery. The management of patients with mild OSA is still evolving. Treatment options for children with mild OSA include watchful waiting, leukotriene inhibitors and/or inhaled nasal corticosteroids, and adenotonsillectomy. Treatment decisions should be made after a frank discussion of the risk and benefits of therapies between the patient, caregivers, and physician. Children with poor quality of life and school performance are candidates for adenotonsillectomy. Close clinical follow-up is necessary for children managed conservatively since a small number of patients, especially those who are obese, can develop more significant obstruction over time. Obese children and those patients with severe disease are at risk for persistent OSA following primary treatment with adenotonsillectomy. Historically, positive airway pressure (PAP) was the mainstay of treatment for persistent disease after adenotonsillectomy. While PAP can be effective in treating children with OSA, adherence is often poor. The utilization of drug-induced sleep endoscopy (DISE) has allowed for surgeons to identify and target additional sites of obstruction. Based on DISE findings, children with residual OSA may be candidates for surgical procedures such as lingual tonsillectomy, pharyngoplasty, supraglottoplasty, or tongue base reduction. 


\section{Introduction}

Obstructive sleep apnea (OSA) is a sleep-related breathing disorder characterized by intermittent episodes of upper airway collapse during sleep. Pediatric OSA affects 2 to $3 \%$ of school-aged children. The pathophysiology of this disorder in children is multifactorial, but two significant risk factors include adenotonsillar hypertrophy and obesity. Pediatric OSA has been linked to metabolic changes, growth inhibition, and cardiovascular sequelae. Furthermore, there is a growing body of literature demonstrating the negative impact of OSA on quality of life (QOL) and cognitive function in children [1]. Pediatric OSA has been associated with behavior problems, poor attention, cognitive deficits, and poor school performance. Thus, the American Academy of Pediatrics now recommends that clinicians screen for OSA during routine health maintenance visits [2•].

Full-night polysomnogram (PSG) is the gold standard for the diagnosis of pediatric OSA. The obstructive apnea hypopnea index (the number of obstructive respiratory events per hour) determines the severity of disease. Children with an obstructive apnea hypopnea index (AHI) greater than 1 are diagnosed with obstructive sleep apnea. According to the most commonly utilized system, an AHI between 1 and 5 indicates mild OSA, while an AHI greater than 10 is diagnostic of severe OSA. Other PSG parameters that may indicate severe obstruction include nadir oxygen saturation less than $80 \%$ and carbon dioxide retention (hypoventilation). Although PSG reliably measures the presence of OSA and provides an objective scale for OSA severity, it fails to quantify the impact of OSA on a child's general wellbeing, including emotional and behavioral health. Thus, when evaluating treatment outcomes for pediatric OSA, it is essential to consider both PSG parameters and QOL assessments.

The primary, initial treatment for moderate to severe OSA in children is adenotonsillectomy. According to surgeon and patient preference, a complete tonsillectomy may be performed verses an intra-capsular tonsillotomy. A variety of techniques for this surgical procedure can be utilized, including monopolar electrocautery, coblation, and microdebridement. Overnight observation is essential for children at risk for perioperative respiratory complications, such as those with obesity, Trisomy 21, or severe pre-operative disease [3].

Even though children with mild OSA do not have significant gas exchange abnormalities noted on PSG, they can suffer significant impairment of behavior, daytime functioning, and family life. Treatment options for mild OSA include observation, anti-inflammatory medications, and adenotonsillectomy (Fig. 1). Intranasal corticosteroids and leukotriene inhibitors are the most commonly utilized anti-inflammatory medications to treat pediatric OSA. Patients with poor QOL may benefit from adenotonsillectomy, while those that are less symptomatic can be observed [4]. Therapy, either medical or surgical, may also be warranted in symptomatic children with sleep-disordered breathing who have not undergone a PSG or in whom the AHI was less than 1 on PSG.

While adenotonsillectomy is the primary treatment for pediatric OSA, cure rates are variable with studies reporting persistent disease in 17 to $73 \%$ of children [5, 6]. Traditionally, children with persistent OSA following adenotonsillectomy were treated with positive airway pressure (PAP) therapy. Many children, however, have difficulty tolerating this therapy and compliance is often poor. With the recent development of drug-induced sleep endoscopy (DISE), surgeons can now identify anatomic sites of upper airway obstruction in children with persistent disease. Surgical therapies such as lingual tonsillectomy, pharyngoplasty, supraglottoplasty, or tongue base reduction have been developed to target these sites of residual obstruction.

\section{Treatment}

- Obesity is a risk factor for OSA. Rates of OSA in obese children range from 13 to $66 \%$, significantly higher than the prevalence of OSA in normal weight children. Obese children are also at risk for persistent obstruction following adenotonsillectomy. 

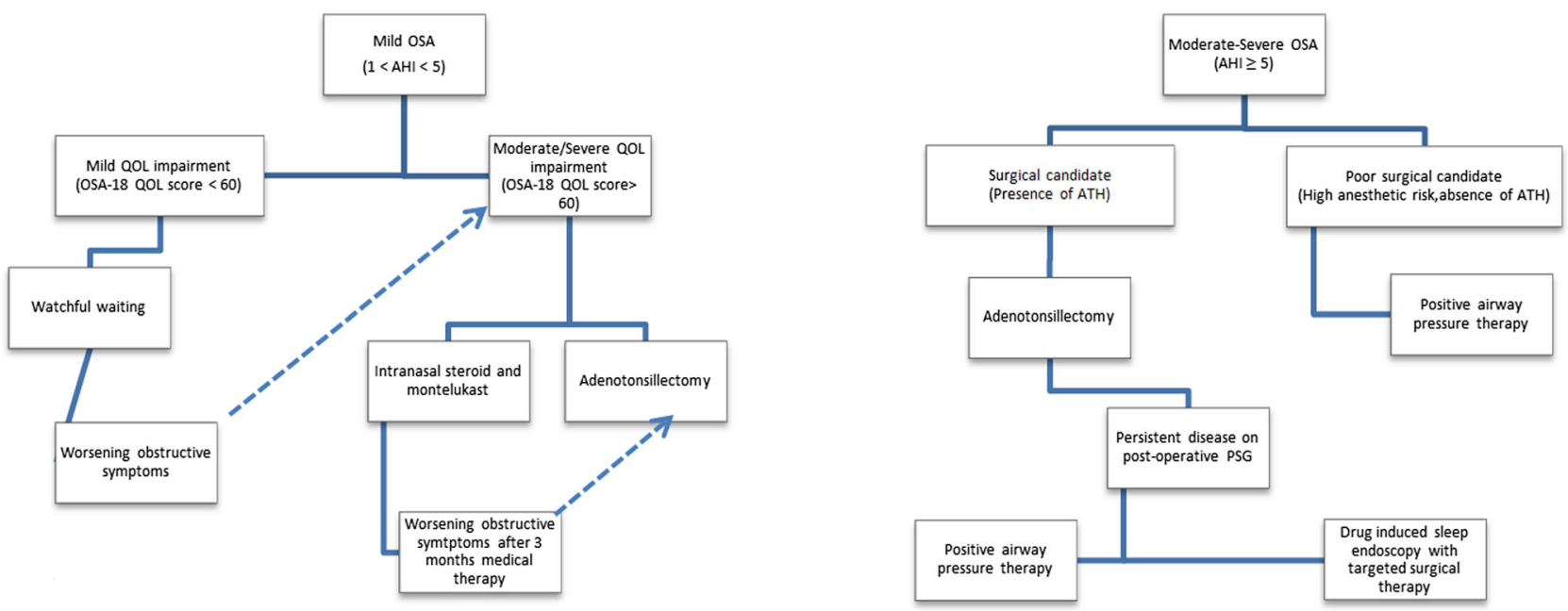

Fig. 1. Treatment algorithm for pediatric obstructive sleep apnea [OSA obstructive sleep apnea, $A H I$ apnea hypopnea index, OSA-18 QOL OSA-18 Quality of Life Questionnaire, ATH adenotonsillar hypertrophy].

- Few studies have evaluated the effect of weight loss on OSA in the pediatric population. Future research is needed to determine the impact of weight loss on OSA outcomes in children and adolescents.

- In an obese adolescent population attending a residential weight loss treatment center, the prevalence of OSA was $39 \%$. Following weight loss, OSA resolved in $71 \%$ of the patients. Adolescents with tonsillar hypertrophy were less likely to achieve resolution of OSA with weight loss [7, Class III].

- A recent review of OSA outcomes in obese adults that underwent bariatric surgery demonstrated improvement in OSA severity in the majority of patients. However, $50 \%$ of subjects had persistent OSA following bariatric surgery [8].

- Multi-disciplinary weight loss programs that feature lifestyle interventions such as behavioral counseling, nutrition education, and scheduled physical activity allow for meaningful weight reduction in obese children and adolescents [9].

- Anti-inflammatory medications have the potential to reduce mucosal inflammation and decrease adenotonsillar hypertrophy in children with OSA.

- Medical therapies, including intranasal corticosteroids and montelukast, are treatment options for children with mild to moderate OSA.

- Treatment with inhaled intranasal corticosteroids, such as fluticasone, budesonide, or mometasone furoate, results in reduction of the $\mathrm{AHI}$ in children with mild OSA [10•, class I]. 
- Montelukast has been utilized to treat both non-severe primary OSA and mild persistent OSA following adenotonsillectomy. Children treated with montelukast demonstrate improvements in obstructive symptoms and PSG parameters [11••; Class I; 12].

- The duration of medical therapy required to treat pediatric OSA is still being investigated with the majority of studies reporting at least 3 months of treatment.

\section{Intranasal corticosteroids}

Mometasone furoate (intranasal)

Several studies have demonstrated improvements in OSA severity in children treated with inhaled intranasal corticosteroids. The authors hypothesize that intranasal corticosteroids reduce the disease burden of OSA by decreasing upper airway resistance and mucosal inflammation. In addition, intranasal corticosteroids also control symptoms of allergic rhinitis, a common co-morbid medical condition in children with OSA. Recently, a randomized controlled trial was conducted to assess the efficacy of mometasone furoate in the treatment of mild OSA [10•, class I]. Children between the ages of 6 and 18 years were randomized to receive either two sprays per nostril of $50 \mathrm{mcg}$ of mometasone furoate or placebo for 4 months. The AHI on PSG in the mometasone furoate group decreased from 2.7 to 1.7 while the AHI in the placebo group increased from 2.5 to 2.9. The main side effect of intranasal corticosteroids is epistaxis. The safety and effectiveness of mometasone furoate have not been investigated in children less than 2 years of age.

\section{Leukotriene inhibitors}

Leukotrienes are inflammatory mediators of the respiratory tract that have been implicated in the pathogenesis of both asthma and allergy. Recently, researchers have been studying the role of leukotrienes in the pathophysiology of adenotonsillar hypertrophy and OSA. Increased expression of leukotriene receptors have now been identified in the tonsillar tissue of children with OSA [13]. Thus, researchers have begun to investigate the role of montelukast, a leukotriene inhibitor, in the treatment of pediatric OSA [14].

Goldbart et al. conducted a double blind, placebo-controlled study to assess whether children with OSA would benefit from treatment with montelukast $[11 \bullet \bullet$, class I]. Children ages 2 to 10 years old with non-severe OSA (AHI between 1 and 10 on PSG) were randomized to receive either montelukast or placebo for 12 weeks. In the montelukast group, the mean obstructive apnea index significantly improved from 3.7 to 1.9, while there was no change in the mean apnea index of the placebo group (3.5 to 3.7). The adenoid size on lateral neck radiograph also significantly decreased in the montelukast group. In a large retrospective review of 836 children with mild OSA (AHI between 1 and 5 on PSG), over half of children had 
resolution of their OSA following 3 months of montelukast therapy. However, approximately $20 \%$ of children had either no response or worsening of obstruction following medical therapy and required adenotonsillectomy. Obesity and age greater than 7 years old predicted a poor response to montelukast [15; Class IV].

Montelukast is now available as a generic. The medication can be dispensed as a chewable tab or as granules. The typical dose is $4 \mathrm{mg}$ for children 2 to 6 years of age and $5 \mathrm{mg}$ for children 6 to 14 years of age. Side effects are uncommon but may include behavioral problems and neuropsychiatric events. Studies have failed to identify a definitive link between leukotriene inhibitors and suicide [16].

\section{Positive airway pressure}

- The aim of positive airway pressure (PAP) therapy is to prevent obstruction by mechanically stenting open the upper airway using an air compressor attached to a facemask.

- PAP is effective in improving both AHI and nadir oxygen saturation in children with obstructive disease [17; Class III].

- PAP is used primarily in children who have persistent OSA following surgical intervention. PAP is also an option for children that are poor surgical candidates.

- PAP is effective in treating children with hypoventilation associated with underlying conditions such as obesity and muscular dystrophy.

- The main limitation of PAP therapy in children is poor compliance.

Continuous positive airway pressure and bilevel positive airway pressure

PAP therapy consists of three main components: (1) a machine with an air pump that delivers positive pressure, (2) a mask that fits over the mouth and/or nose, and (3) tubing to connect the machine and mask. The two most common types of PAP therapy utilized in children are continuous positive airway pressure (CPAP) and bilevel positive airway pressure (BiPAP). cPAP features continuous positive airway pressure, while BiPAP delivers preset inspiratory positive airway pressure during inspiration and expiratory positive airway pressure during expiration. BiPAP is also referred to as non-invasive positive pressure ventilation and is utilized to treat children with hypoventilation.

Traditionally, children being considered for PAP therapy are scheduled for an overnight titration study in the sleep laboratory to determine the optimal PAP settings. Prior to the study, it is essential that children be fitted with an appropriately sized mask that maximizes comfort and minimizes air leak. The two types of masks typically used are nasal and full-face masks. To prevent aspiration, children should only be fit with full-face masks if they have the dexterity to remove the mask. During the overnight titration PSG, pressures are gradually increased until obstructive events and desaturations are reduced. An adequate titration study identifies the lowest pressure that eliminates obstruction in supine rapid eye movement sleep. 
Titration studies are still utilized for children with significant co-morbid conditions, severe sleep apnea, and hypoventilation. However, auto cPAP is being used with increasing frequency in pediatric patients [18]. An auto CPAP device automatically adjusts the level of pressure delivered according to the patient's amount of obstruction. Auto CPAP allows providers to initiate PAP therapy as soon as OSA is diagnosed and thus eliminates the delay in treatment that results from waiting for a formal titration study. An additional advantage of the variable pressure response of auto CPAP is the capability to adjust to situations such as upper airway infections, different sleeping positions, and changes in weight. Auto CPAP has the potential to improve compliance in children by achieving the same treatment efficacy with the lowest possible pressure.

While PAP therapy is effective in treating OSA in children [19], widespread use is limited because of poor compliance. Close follow-up is essential to identify problems and improve PAP adherence. Patients should be re-evaluated 30 days after initiating PAP therapy. Objective data regarding compliance should be obtained by downloading usage data from the device itself [17, class III]. Behavioral therapy, including desensitization, may improve compliance in some pediatric patients [20]. Common complications of PAP therapy include dry eyes and skin irritation from the mask. There is also concern that long-term use of PAP therapy in children may result in changes in midfacial growth.

- Adenotonsillectomy is the first-line treatment for children with moderate to severe OSA and adenotonsillar hypertrophy [2•].

- Adenotonsillectomy improves quality of life and behavior in children with OSA [1]. Surgery also results in significant improvements in PSG parameters such as the AHI and nadir oxygen saturation.

- A recent study demonstrated that neurocognitive outcomes were similar in children with mild OSA who underwent adenotonsillectomy

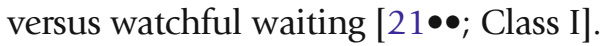

- Patients at high risk for perioperative respiratory complications should be observed overnight in the hospital following adenotonsillectomy [3].

- Non-narcotic medications, such as ibuprofen, are the safest choice for analgesia in children with OSA. Providers should avoid using Tylenol with codeine to treat pain in children following adenotonsillectomy.

Adenotonsillectomy is the surgical removal of the adenoids and palatine tonsils, lymphoid tissue commonly found in the upper airway of children. Adenotonsillectomy is the mainstay of treatment in children with OSA and adenotonsillar enlargement. Recently published guidelines recommend that children with severe co-morbid medical conditions such as obesity, sickle cell disease, and Trisomy 21 undergo PSG prior to surgical therapy [3]. These children are at increased risk for perioperative compilations. 
Thus, PSG confirms the diagnosis of OSA and provides important information about risk stratification. In healthy children with adenotonsillar hypertrophy and significant obstructive symptoms, PSG prior to surgery is not required.

Previous studies have demonstrated improvements in PSG parameters, behavior, and QOL following adenotonsillectomy in children with OSA [1]. However, this data regarding the efficacy of adenotonsillectomy was characterized by a significant limitation and lack of a control group. The recent Childhood Adenotonsillectomy Trial (CHAT) was designed to ad-

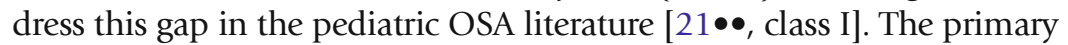
objective of the CHAT was to assess polysomnographic, cognitive, behavioral, and health outcomes in children undergoing treatment for OSA. In this multicenter, prospective study, children with non-severe OSA (AHI $<30$ and nadir oxygen $>90 \%$ for greater than $98 \%$ of total sleep time) were randomized to either early adenotonsillectomy or watchful waiting. The children in the adenotonsillectomy group experienced significant improvements in AHI, quality of life, and behavior compared to the watchful waiting group. Interestingly, though, approximately half of the children in the watchful waiting group had normalization of their PSG after 7 months without any intervention. Objective neurocognitive assessments of attention and executive function were similar in the two groups at the 7 month follow-up. There were several limitations of the CHAT that merit discussion. First; the subjects included in the study were between 5 and 9 years of age. Thus, the findings may not be applicable to younger patients with OSA. In addition, children with attention deficit hyperactivity disorder (ADHD), a common co-morbid condition in children with OSA, were excluded from this study. The CHAT authors concluded that while early adenotonsillectomy has beneficial effects for children with non-severe OSA, watchful waiting is another therapeutic option. The recently published findings of another prospective study comparing adenotonsillectomy and watchful waiting in children with mild OSA were similar [4]. Volsky et al. reported significant improvements in quality of life in the

adenotonsillectomy group compared to the watchful waiting group after 4 months. However, at the 8-month follow-up, while improvements in quality of life scores in the adenotonsillectomy group were maintained, significant improvement in quality of life was also evident in the watchful waiting group.

Adenotonsillectomy is performed under general anesthesia utilizing a variety of techniques. Adenoidectomy is typically performed with the use of a curette, microdebrider, or suction electrocautery. A small amount of adenoid tissue is maintained inferiorly to prevent velopharyngeal insufficiency. Thus, children, especially those less than 2 years of age at the time of initial surgery, are at risk for adenoid regrowth. There are two primary techniques for removing palatine tonsils in children, a complete tonsillectomy and an intra-capsular tonsillotomy. The intra-capsular tonsillotomy spares the tonsillar capsule and is commonly performed with microdebrider or coblation. Complete tonsillectomy removes the entire palatine tonsil using monopolar electrocautery, coblation, cold steel, or bipolar cautery. Proponents of the intra-capsular tonsillotomy technique report decreased use of pain medication and faster return to normal diet 
and activity compared to complete tonsillectomy [22]. In one study, microdebrider intra-capsular tonsillotomy was associated with a decreased risk of postoperative hemorrhage [23]. One of the primary concerns regarding the intra-capsular tonsillotomy technique is tonsillar regrowth. A recent study published on the use of drug-induced sleep endoscopy in children with OSA demonstrated significant obstruction at the level of the lateral pharyngeal wall in patients with small tonsils [24•]. This finding raises concerns that even a small amount of residual tonsillar tissue can cause obstruction in some children. When surgical time and equipment costs are taken into account, the cost of monopolar cautery adenotonsillectomy is significantly less than other techniques [25; Class IV].

Pain and dehydration can occur following adenotonsillectomy. Rates of post-tonsillectomy hemorrhage typically range from 1 to $2 \%$. Perioperative respiratory complications are also common in children with OSA undergoing adenotonsillectomy. Thus, providers that perform adenotonsillectomy in children must be familiar with current recommendations regarding peri-operative management. Recent guidelines published by the American Academy of Otolaryngology-Head and Neck Surgery recommend that the following patients should be observed overnight in the hospital following adenotonsillectomy: (1) children with significant comorbidities such as Trisomy 21 or neuromuscular disorders, (2) children less than 3 years of age, and (3) children with severe OSA (defined as AHI $>10$ or oxygen saturation nadir $<80 \%$ ) [3]. Similarly, the American Academy of Pediatrics recommends overnight observation in the hospital setting for patients with severe OSA (AHI $>24$ or nadir oxygen $<80 \%$ or peak $\mathrm{CO} 2>60 \mathrm{mmHg}$ ), cardiac complications, failure to thrive, obesity, craniofacial anomalies, neuromuscular disorders, or concurrent respiratory infection [2•]. A recent observational cohort study conducted at a tertiary children's hospital aimed to prospectively assess perioperative complications following adenotonsillectomy [26•, class II]. Children less than 3 years of age and those with failure to thrive were more likely to experience major respiratory complications. The authors also reported that abnormalities in gas exchange, specifically nadir oxygen saturation less than $80 \%$ or end-tidal CO2 peak was $>60 \mathrm{mmHg}$, are better predictors of perioperative respiratory complications than AHI values.

Weight gain following adenotonsillectomy has recently been a topic of debate in the otolaryngology literature. A retrospective case control series found that after adenotonsillectomy, obese patients have significant weight gain when compared to control subjects [27, class III]. Similar findings were reported in the CHAT cohort, with children in the adenotonsillectomy group showing an increase in BMI compared to the watchful waiting group. Overweight and obese children were more likely than normal weight children to gain weight after surgery $[21 \bullet \bullet]$. Such findings highlight the importance of weight monitoring and nutritional counseling in children being considered for adenotonsillectomy.

Pain control following adenotonsillectomy can be very challenging. Children with OSA are prone to obstruction when treated with narcotic pain medication. Traditionally, post-adenotonsillectomy pain was treated with Tylenol with codeine. However, after reports of several deaths related to codeine administration following adenotonsillectomy, the Federal Drug 
Enforcement Agency (FDA) issued a "black box warning" for the use of codeine in this setting [28]. Many otolaryngologists are now prescribing hydrocodone with acetaminophen to treat post-

tonsillectomy pain. However, the potential for respiratory depression exists with the utilization of any narcotic in children with severe

obstruction. The optimal post-adenotonsillectomy pain control regimen for children with OSA is still being investigated. A recent metaanalysis found that utilization of non-steroidal anti-inflammatory pain medications (with the exception of ketorolac) in the postoperative period are not associated with significantly increased rates of tonsillar bleeding [29•, class I]. Ibuprofen and acetaminophen are being increasingly utilized for post-tonsillectomy pain, especially in children with severe OSA.

\section{Expansion sphincter phanngoplasty}

Expansion sphincter pharyngoplasty is a palatal procedure that addresses lateral pharyngeal wall collapse identified on drug-induced sleep endoscopy. The surgery was first described in adults but has recently been modified for use in children with severe OSA. Following tonsillectomy, the palatopharyngeus muscles are transected between the upper third and middle third. The cut muscle edge is then pulled superoanterolaterally into a tunnel created in the soft palate and sutured in place. Potential complications include dysphagia and velopharyngeal insufficiency. In a recent study of children with severe OSA, modified expansion sphincter pharyngoplasty resulted in significantly improved cure rates compared to traditional adenotonsillectomy [30]. Children greater than 3 years of age with severe OSA (AHI>20) and significant lateral pharyngeal wall collapse on sleep endoscopy are ideal candidates for this procedure.

\section{Orthodontic treatment}

- Rapid maxillary expansion is a treatment option for mild OSA in older children and adolescents with malocclusion [31].

- Mandibular advancement appliances improve AHI and nadir oxygen saturation, but long-term efficacy has not been established [32, 33].

- Maxillomandibular advancement, while rarely performed in children, is a surgical option for adolescents with severe OSA and craniofacial anomalies such as micrognathia [34].

The aim of rapid maxillary expansion is to widen the maxillary bone by distraction osteogenesis. Older children and adolescents with mild OSA that do not have significant tonsillar hypertrophy but do have dental malocclusion may be candidates for this therapy. Rapid maxillary expansion is best accomplished in patients with a narrow palate and associated deep bite, retrusive bite, or crossbite. 
Appropriately selected patients undergoing rapid maxillary expansion were noted to have improvements in AHI on post-treatment PSG [31, 35]

Oral appliances to reposition the mandible are commonly used to treat adults with mild to moderate OSA, but the utility of such devices has not been extensively evaluated in children. Mandibular advancement appliances (MAA) can increase the lateral dimension of the velopharyngeal airway as a result of forward positioning of the mandible and also reduce collapsibility of the airway. A recent systematic review of MMA use in children with OSA demonstrated improvements in AHI but failure to normalize PSG parameters [32]. The studies regarding MMA feature small sample sizes and lack of control groups. Furthermore, the long-term impact of MAA on dental alveolar anatomy and mandibular growth is unknown.

\section{Surgical treatment for persistent OSA}

- While adenotonsillectomy is the primary treatment for pediatric OSA, cure rates are variable with studies reporting persistent disease in 17 to $73 \%$ of children $[5,6]$.

- Drug-induced sleep endoscopy (DISE) can identify additional sites of obstruction in patients with persistent OSA after adenotonsillectomy and direct further surgical treatment [36, class IV].

- Lingual tonsil hypertrophy and occult laryngomalacia are two important causes of persistent OSA in children that have previously undergone adenotonsillectomy [37; Class IV].

- When tongue base collapse is noted on DISE, techniques such as tongue base suspension [38] and submucosal tongue base reduction [39] can be used to treat OSA in children.

Drug-induced sleep endoscopy

Drug-induced sleep endoscopy (DISE) can identify the level of airway obstruction in patients with persistent OSA. In children, DISE, performed with a flexible nasal endoscope, is accomplished while the patient is sedated and breathing spontaneously. Typical agents used for DISE in children are propofol, ketamine, or dexmedetomidine. In a recent study examining the feasibility of DISE in children with persistent OSA, the majority of subjects had multilevel obstruction [40]. DISE is increasingly being utilized to direct surgical therapy in children with residual OSA. Future research is needed to determine whether DISE improves surgical outcomes in these children. 
adenotonsillectomy. Resection of lingual tonsils is typically performed under endoscopic visualization using coblation. Other techniques for lingual tonsillectomy include utilization of the microdebrider, electrocautery, or carbon dioxide laser. Lingual tonsillectomy results in improvement of PSG indices, including the respiratory disturbance index, in patients with persistent OSA [41, class II]. Multi-level, single-stage surgery in children undergoing lingual tonsillectomy should be avoided due to the risk of oropharyngeal stenosis [42; Class III].

Younger children with co-morbid medical conditions are more likely to have occult laryngomalacia identified as the cause of persistent obstruction [37, class IV] on DISE. In a recent retrospective review of 22 children with OSA and occult laryngomalacia, supraglottoplasty resulted in significant improvement in the AHI [43].

Tongue base collapse is another cause of residual obstruction in children with persistent OSA. Techniques used to address tongue base obstruction in children include tongue base suspension and tongue base reduction. Wooten and Shott published a review of 31 children with persistent OSA treated with tongue base suspension and radiofrequency ablation of the tongue base. Following the procedure, the mean AHI improved from 14.1 to 6.4 events per hour [44].

\section{Compliance with Ethics Guidelines}

\section{Conflict of Interest}

No potential conflicts of interest relevant to this article were reported.

\section{Human and Animal Rights and Informed Consent}

This article does not contain any studies with human or animal subjects performed by any of the authors.

\section{References and Recommended Reading}

Papers of particular interest, published recently, have been highlighted as:

- Of importance

- Of major importance

1. Baldassari CM et al. Pediatric obstructive sleep apnea and quality of life: a meta-analysis. Otolaryngol Head Neck Surg. 2008;138(3):265-73.

2. Marcus CL et al. Diagnosis and management of childhood obstructive sleep apnea syndrome. Pediatrics. 2012;130(3):e714-55.

Offers evidence-based guidelines to help providers determine which children require polysomnogram prior to adenotonsillectomy.
3. Roland PS et al. Clinical practice guideline: polysomnography for sleep-disordered breathing prior to tonsillectomy in children. Otolaryngol Head Neck Surg. 2011;145(1 Suppl):S1-15.

4. Volsky PG et al. Adenotonsillectomy vs observation for management of mild obstructive sleep apnea in children. Otolaryngol Head Neck Surg. 2014;150(1):12632.

5. Bhattacharjee R et al. Adenotonsillectomy outcomes in treatment of obstructive sleep apnea in children: a 
multicenter retrospective study. Am J Respir Crit Care Med. 2010;182(5):676-83.

6. Brietzke SE, Gallagher D. The effectiveness of tonsillectomy and adenoidectomy in the treatment of pediatric obstructive sleep apnea/hypopnea syndrome: a meta-analysis. Otolaryngol Head Neck Surg. 2006;134(6):979-84.

7. Van Hoorenbeeck K et al. Weight loss and sleepdisordered breathing in childhood obesity: effects on inflammation and uric acid. Obesity (Silver Spring). 2012;20(1):172-7.

8. Sarkhosh K et al. The impact of bariatric surgery on obstructive sleep apnea: a systematic review. Obes Surg. 2013;23(3):414-23.

9. Oude Luttikhuis H. et al. Interventions for treating obesity in children. Cochrane Database Syst Rev 2009;(1): p. CD001872.

10. Chan CC et al. Intranasal corticosteroids for mild childhood obstructive sleep apnea-a randomized; placebocontrolled study. Sleep Med. 2015;16(3):358-63.

Idenitified intranasal corticosteroids as a useful treatment adjunct in patients with mild OSA; specifically those suffering from allergic rhinitis.

11.• Goldbart AD, Greenberg-Dotan S, Tal A. Montelukast for children with obstructive sleep apnea: a doubleblind; placebo-controlled study. Pediatrics. 2012;130(3):e575-80.

Established montelukast as a treatment option for children with mild OSA.

12. Kheirandish L, Goldbart AD, Gozal D. Intranasal steroids and oral leukotriene modifier therapy in residual sleep-disordered breathing after tonsillectomy and adenoidectomy in children. Pediatrics.

2006;117(1):e61-6.

13. Tsaoussoglou $\mathrm{M}$ et al. Cysteinyl leukotriene receptors in tonsillar B- and T-lymphocytes from children with obstructive sleep apnea. Sleep Med. 2012;13(7):87985.

14. Goldbart AD et al. Leukotriene modifier therapy for mild sleep-disordered breathing in children. Am J Respir Crit Care Med. 2005;172(3):364-70.

15. Kheirandish-Gozal L et al. Antiinflammatory therapy outcomes for mild OSA in children. Chest. 2014;146(1):88-95.

16. Schumock GT et al. Association between leukotrienemodifying agents and suicide: what is the evidence? Drug Saf. 2011;34(7):533-44.

17. Marcus CL et al. Adherence to and effectiveness of positive airway pressure therapy in children with obstructive sleep apnea. Pediatrics. 2006;117(3):e44251.

18. Palombini L, Pelayo R, Guilleminault C. Efficacy of automated continuous positive airway pressure in children with sleep-related breathing disorders in an attended setting. Pediatrics. 2004;113(5):e412-7.

19. Marcus CL et al. Effects of positive airway pressure therapy on neurobehavioral outcomes in children with obstructive sleep apnea. Am J Respir Crit Care Med. 2012;185(9):998-1003.
20. Rains JC. Treatment of obstructive sleep apnea in pediatric patients. Behavioral intervention for compliance with nasal continuous positive airway pressure. Clin Pediatr (Phila). 1995;34(10):535-41.

21.• Marcus CL et al. A randomized trial of adenotonsillectomy for childhood sleep apnea. N Engl J Med. 2013;368(25):2366-76.

First randomized trial to assess adenotonsilectomy. Highlighted the benefits of adenotonsillectomy; including improvments in PSG indices and quality of life measures; in children with mild OSA. Watchful waiting was identified as a reasonable alternative to surgery.

22. Wilson YL, Merer DM, Moscatello AL. Comparison of three common tonsillectomy techniques: a prospective randomized; double-blinded clinical study. Laryngoscope. 2009;119(1):162-70.

23. Gallagher TQ et al. Analyzing factors associated with major complications after adenotonsillectomy in 4776 patients: comparing three tonsillectomy techniques. Otolaryngol Head Neck Surg. 2010;142(6):886-92.

24. Ulualp SO, Szmuk P. Drug-induced sleep endoscopy for upper airway evaluation in children with obstructive sleep apnea. Laryngoscope. 2013;123(1):292-7.

Described the technique of DISE and identified common sites of obstruction in children with OSA.

25. Thottam PJ et al. The utility of common surgical instruments for pediatric adenotonsillectomy. Laryngoscope. 2015;125(2):475-9.

26. Thongyam A et al. Predictors of perioperative complications in higher risk children after adenotonsillectomy for obstructive sleep apnea: a prospective study.

Otolaryngol Head Neck Surg. 2014;151(6):1046-54.

First prospective study to evaluate risk factors for perioperative complications in children with OSA undergoing

adenotonsillectomy.

27. Lewis TL et al. Weight gain after adenotonsillectomy: a case control study. Otolaryngol Head Neck Surg. 2015;152(4):734-9.

28. Kelly LE et al. More codeine fatalities after tonsillectomy in North American children. Pediatrics. 2012;129(5):e1343-7.

29. $\quad$ Riggin L et al. A 2013 updated systematic review \& meta-analysis of 36 randomized controlled trials; no apparent effects of non steroidal anti-inflammatory agents on the risk of bleeding after tonsillectomy. Clin Otolaryngol. 2013;38(2):115-29.

Meta-analysis detailing the safety of non steroidal anti-in-

flammatory use after adenotonsillectomy.

30. Ulualp SO. Modified expansion sphincter pharyngoplasty for treatment of children with obstructive sleep apnea. JAMA Otolaryngol Head Neck Surg. 2014;140(9):817-22.

31. Villa MP et al. Efficacy of rapid maxillary expansion in children with obstructive sleep apnea syndrome: 36 months of follow-up. Sleep Breath. 2011;15(2):179-84.

32. Nazarali N. et al. Mandibular advancement appliances for the treatment of pediatric obstructive sleep apnea: a systematic review. Eur J Orthod. 2015. doi:10.1093/ ejo/cju101. 
33. Zhang C, He H, Ngan P. Effects of twin block appliance on obstructive sleep apnea in children: a preliminary study. Sleep Breath. 2013;17(4):1309-14.

34. Bell RB, Turvey TA. Skeletal advancement for the treatment of obstructive sleep apnea in children. Cleft Palate Craniofac J. 2001;38:147-54.

35. Villa MP et al. Rapid maxillary expansion in children with obstructive sleep apnea syndrome: 12-month follow-up. Sleep Med. 2007;8(2):128-34.

36. Wootten CT, Chinnadurai S, Goudy SL. Beyond adenotonsillectomy: outcomes of sleep endoscopydirected treatments in pediatric obstructive sleep apnea. Int J Pediatr Otorhinolaryngol. 2014;78(7):115862.

37. Chan DK, Jan TA, Koltai PJ. Effect of obesity and medical comorbidities on outcomes after adjunct surgery for obstructive sleep apnea in cases of adenotonsillectomy failure. Arch Otolaryngol Head Neck Surg. 2012;138(10):891-6.

38. Hartzell LD et al. Tongue base suspension in children with cerebral palsy and obstructive sleep apnea. Int J Pediatr Otorhinolaryngol. 2013;77(4):534-7.
39. Maturo SC, Mair EA. Submucosal minimally invasive lingual excision: an effective; novel surgery for pediatric tongue base reduction. Ann Otol Rhinol Laryngol. 2006;115(8):624-30.

40. Durr ML et al. Drug-induced sleep endoscopy in persistent pediatric sleep-disordered breathing after adenotonsillectomy. Arch Otolaryngol Head Neck Surg. 2012;138(7):638-43.

41. Kuo CY, Parikh SR. Can lingual tonsillectomy improve persistent pediatric obstructive sleep apnea? Laryngoscope. 2014;124(10):2211-2.

42. Prager JD et al. Oropharyngeal stenosis: a complication of multilevel; single-stage upper airway surgery in children. Arch Otolaryngol Head Neck Surg. 2010;136(11):1111-5.

43. Chan DK, Truong MT, Koltai PJ. Supraglottoplasty for occult laryngomalacia to improve obstructive sleep apnea syndrome. Arch Otolaryngol Head Neck Surg. 2012;138(1):50-4.

44. Wootten CT, Shott SR. Evolving therapies to treat retroglossal and base-of-tongue obstruction in pediatric obstructive sleep apnea. Arch Otolaryngol Head Neck Surg. 2010;136(10):983-7. 